\title{
Random Tilings of High Symmetry: II. Boundary Conditions and Numerical Studies
}

\author{
M. Widom, ${ }^{1}$ N. Destainville, ${ }^{2}$ R. Mosseri, ${ }^{3}$ and F. Bailly ${ }^{4}$
}

Received December 11, 2004; accepted April 29, 2005

\begin{abstract}
We perform numerical studies including Monte Carlo simulations of high rotational symmetry random tilings. For computational convenience, our tilings obey fixed boundary conditions in regular polygons. Such tilings are put in correspondence with algorithms for sorting lists in computer science. We obtain statistics on path counting and vertex coordination which compare well with predictions of mean-field theory and allow estimation of the configurational entropy, which tends to the value 0.568 per vertex in the limit of continuous symmetry. Tilings with phason strain appear to share the same entropy as unstrained tilings, as predicted by mean-field theory. We consider the thermodynamic limit and argue that the limiting fixed boundary entropy equals the limiting free boundary entropy, although these differ for finite rotational symmetry.
\end{abstract}

KEY WORDS: Random tiling; Monte Carlo; quasicrystal; entropy.

\section{INTRODUCTION}

A tiling is a filling, without gaps or overlaps, of a given region of a $d$ dimensional Euclidean space, with tiles which differ according to their shapes, sizes, and orientations. In the present paper, the tiles are $d$ dimensional rhombohedra, which we will generically call "rhombi" in the following. Each tile is the projection of a $d$-dimensional face of a $D$

\footnotetext{
${ }^{1}$ Department of Physics, Carnegie Mellon University, Pittsburgh, PA 15213, USA; e-mail: widom@andrew.cmu.edu

${ }^{2}$ Laboratoire de Physique Théorique, UMR CNRS-UPS 5152, Université Paul Sabatier, 31062 Toulouse Cedex 04, France.

${ }^{3}$ Laboratoire de Physique Théorique des Liquides, Tour 24, Boîte 121, 4, Place Jussieu, 75252 Paris Cedex 05, France.

${ }^{4}$ LPSB-CNRS, 92195 Meudon Cedex, France.
} 
dimensional hypercube $(D>d)$ into $d$-dimensional space. The difference $D-d$ is known as the codimension of the tiling, and we say that we are dealing with $D \rightarrow d$ tilings. Details of this construction can be found in a previous paper, ${ }^{(1)}$ hereafter referred to as "paper I". We mainly focus on the case $d=2$. A random rhombus tiling can be viewed as a fluctuating membrane in this higher dimensional space, ${ }^{(2)}$ the membrane being the union of hypercube faces (see paper $I^{(1)}$, Section 2.1).

Rhombus tilings are dual to de Bruijn grids. ${ }^{(1,3)}$ In two dimensions, these grid lines pass through the midpoints of parallel rhombus edges. Every rhombus edge orientation defines a family of effectively parallel de Bruijn grid lines. De Bruijn grid lines within a family never cross. In contrast, lines of different families $d o$ cross, and their crossing defines a rhombus of the tiling. There are $D$ rhombus edge orientations and hence $D$ families of de Bruijn grid lines.

Rhombus tilings provide simple models for quasicrystals, $(4,5)$ metal alloys that exhibit rotational symmetry forbidden by conventional crystallography. One of the key properties of random tilings ${ }^{(6)}$ is their configurational entropy that may play a role in stabilizing the quasicrystal state. The source of entropy is localized tile rearrangements known as phason flips. Groups of three adjacent rhombi may be permuted so that their perimeter remains fixed while their shared vertex moves to a nearby point. Our present focus, as in paper I, is the limit of high rotational symmetry obtained as $D \rightarrow \infty$ at fixed $d$. We examine this limit because of its intrinsic interest and because it deepens our understanding of finite $D$ tiling models relevant for real alloy systems.

As usual in statistical mechanics, tiling systems can have different boundary conditions, such as free, fixed or periodic. For example, the tilings in Figs. 1, 2 or 14, have fixed polygonal boundaries. It is believed that free and periodic boundary tilings reach equal entropies at the thermodynamic limit. Fixed boundary tilings, in contrast, exhibit entropies that are strictly smaller than the free boundary entropy. For example, the fixed hexagonal-boundary entropy of $3 \rightarrow 2$ tilings (see Fig. 1, left) equals $0.261{ }^{(7)}$ while the free boundary entropy equals 0.323 . $^{\left({ }^{2}\right)}$ This phenomenon can be understood ${ }^{(7,9-11)}$ by inspection of Fig. 1. The local entropy density displays a gradient between crystalline regions near the boundary, where the entropy density vanishes, and the central region, where the entropy density reaches a maximum value equal to the free boundary quasicrystalline value. Only at the very center of the tiling does the influence of the boundary disappear.

Paper $I^{(1)}$ developed a mean-field theory for free boundary tilings, applicable to the limit of high rotational symmetry. Two earlier papers ${ }^{(12,13)}$ presented initial studies of this problem. The first paper $^{(12)}$ proposed an 

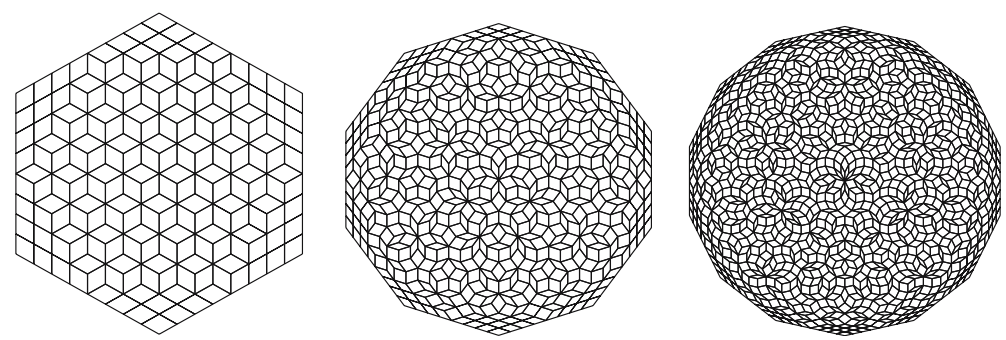

Fig. 1. Fixed boundary $D \rightarrow 2$ tilings with $D=3,5$ and 7 . The side lengths of the polygons are all $p=8$.

upper bond on the entropy in the limit of large $D$ and discussed problems associated with the thermodynamic limit of tiling models. The second one $^{(13)}$ presented a preliminary mean-field approach of the entropy calculation. In the present paper we focus on fixed boundary tilings because they are easy to simulate numerically. We present results of our simulations, including an accurate estimate of the limiting entropy density. We investigate the role of fixed boundary conditions and argue for a boundarycondition-independent thermodynamic limit in the limit $D \rightarrow \infty$. Similarly, we show that phason strain does not influence the entropy for large $D$.

The organization of this paper is as follows. We start in Section 2 with a discussion of fixed boundary tilings, and describe their relationship to interesting problems of pure mathematics. Next, we review the problem of the thermodynamic limit in Section 3 where we argue that free and fixed boundary tilings attain the same thermodynamic limit as $D \rightarrow \infty$. We also explore finite $D$ corrections to the fixed boundary entropy and their relation to the inhomogeneity of the tilings. Then, Section 4 describes our Monte Carlo simulations. In that section we explore the entropy, path counting statistics, vertex coordination statistics and the role of phason strain. We also confirm numerically the results of Section 3. The paper is written so that the reader does not need the notations of paper I, except in the appendices.

\section{FIXED BOUNDARY TILINGS}

Fixed boundary tilings such as those of Fig. 1 possess the most natural boundary conditions for tilings coded by combinatorial objects such as generalized partitions or sorting algorithms. Even though these boundary conditions are unnatural for bulk quasicrystals, this inconvenience is counter-balanced by the powerful tools provided by such codings in terms of understanding of tiling set structures and enumeration of tilings. 
Furthermore, we will demonstrate in this paper that fixed and free boundary tilings become equivalent in the large codimension limit, which will justify a posteriori the use of fixed boundary conditions.

As already described in the introduction, such fixed boundary tilings lack a proper thermodynamic limit for finite $D$ because the boundary has a strong macroscopic effect on the whole tiling. ${ }^{(7,9-11,14)}$ This leads to a spectacular effect known as the "arctic circle phenomenon"(10) in hexagonal $(D=3)$ tilings, where the tiling is periodic (and "frozen") outside a perfect circle at the large size limit and random inside this circle. More generally and for larger $D$, fixed boundary tilings have an effective codimension that is smaller near the boundary than in the bulk. Very near the boundary the effective codimension vanishes. The tiling becomes a crystalline domain comprising a single tile type. The local entropy density vanishes because no phason flips are possible. In the membrane picture, this region corresponds to a flat area with large tilt. Further from the boundary the effective codimension grows. The variable codimension results in an entropy density gradient, growing from zero at the boundary to the maximal, free boundary, entropy density at the tiling center. Consequently, fixed boundary tilings have a smaller total entropy per tile than free boundary tilings.

In contrast, we will show in Section 3 that when $D$ becomes large (Fig. 2), this heterogeneity diminishes and a thermodynamic limit is restored. Since fixed boundary tilings are easier to specify and manipulate for arbitrary $D$, they are the appropriate tool to tackle large $D$ entropies or other statistical properties. In the following, the fixed boundary

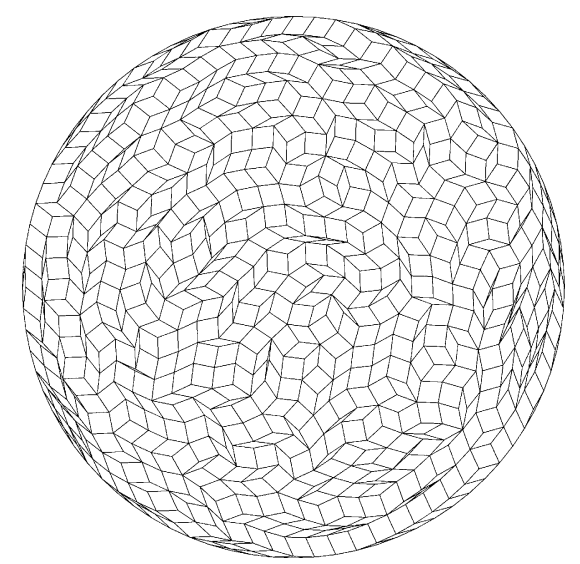

Fig. 2. A $40 \rightarrow 2$ fixed boundary tiling with one de Bruijn line in each family. 
entropies per tile will be denoted by $\bar{\sigma}_{D}$ and their limiting value by $\bar{\sigma}_{\infty}$. Corresponding values without the over-bar refer to free boundary tilings.

In terms of de Bruijn dualization, any two lines of two different families intersect inside a fixed boundary tiling ${ }^{(15)}$ (the grid is said to be "complete"). Duality associates tile with the intersection of two lines. Therefore if there are $k_{i}$ lines in each family $F_{i}$, the number of tiles is

$$
N_{T}=\sum_{1 \leqslant i<j \leqslant D} k_{i} k_{j}
$$

If all $k_{i}$ are set to $1, N_{T}=D(D-1) / 2$.

The relationship between tilings and partitions has been widely explored in references refs. 7, 15-17. The idea is to code a random rhombus tiling by an array of integers satisfying certain ordering constraints. Figure 3 illustrates this point in the simple hexagonal case:(7) there is a one-to-one correspondence between hexagonal tilings filling a centrally symmetric hexagon of sides $k, l$ and $p$ on the one hand and sets of integers arranged in a rectangular array $k \times l$, decreasing in each row and each column, on the other hand. These integers are bounded between zero and the side length $p$. They decrease monotonically. Such a set of integers is called a plane partition.

This point of view can be extended to any $D \rightarrow d$ problem $^{(15-17)}$ : any $D \rightarrow d$ tiling can be coded with a generalized partition on an array related to a $D-1 \rightarrow d$ tiling. This correspondence is also one-to-one. However, tilings coded by partitions have fixed polytopal boundary conditions. In

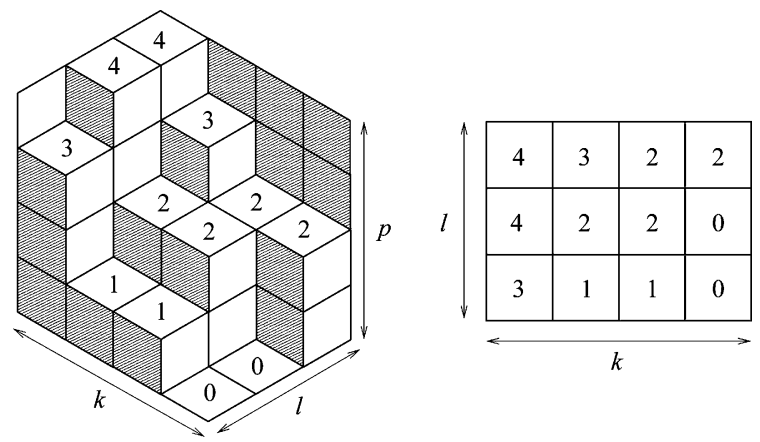

Fig. 3. Three-dimensional representation of an hexagonal tiling filling a centrally symmetric hexagon (left). This object can be seen as a staking of cubes. The height of the different stacks can be arranged in an array (right). They are decreasing in rows and columns. This array together with these order relations constitutes a plane partition problem. 
two dimensions, the boundaries are centrally symmetric $2 D$-gons (see Fig. 1).

We now present an analogy between fixed boundary tilings and algorithms for sorting lists. ${ }^{(15)}$ Although Computer Science is not our motivation, we are interested in the same enumeration problem. ${ }^{(18,19)}$ More generally, the rich topological and combinatorial properties of random tilings make them an active field of research in pure mathematics, combinatorics and computer science. ${ }^{(10,20-22)}$ We shall use results from these fields throughout this paper.

In the sorting language, a comparator $[i ; j]$ acts on a list $\left(x_{1}, x_{2}, \ldots, x_{D}\right)$ of numbers as follows: $x_{i}$ and $x_{j}$ are, respectively, replaced by $\min \left(x_{i}, x_{j}\right)$ and $\max \left(x_{i}, x_{j}\right)$. Following Knuth, ${ }^{(18)}$ we call a complete sorting algorithm a sequence of such comparators which sorts in the increasing order any list of real numbers $\left(x_{1}, x_{2}, \ldots, x_{D}\right)$. This sorting algorithm will be called primitive if each comparator can be written $[i ; i+1]$. We also suppose that this algorithm is not redundant, that is to say it does not contain any comparator $[i ; j]$ that could be suppressed because previous comparators already insure that $x_{i} \leqslant x_{j}$. Knuth shows that a sequence of comparators is a sorting algorithm if it correctly sorts the completely reversed list $(D, D-1, \ldots, 1)$. This means that a complete primitive sorting algorithm is a sequence of comparators $[i ; i+1]$ that transforms the list $(D, D-1$, $\ldots, 1)$ into the list $(1,2, \ldots, D)$.

Such an algorithm has a diagrammatical representation in which the $D$ variables $x_{i}$ are represented by $D$ horizontal lines. Each comparator $[i ; i+1]$ is represented by a crossing between lines $i$ and $i+1$. Figure 4 illustrates this construction. A continuous line follows a number during the sorting process. Since every number must be compared to every other number, and since there is not any redundancy, every line crosses every other line, only once. There are $\left(\begin{array}{l}D \\ 2\end{array}\right)$ crossings.

We now establish the link between sorting algorithms and the de Bruijn representations ${ }^{(1,3)}$ of $D \rightarrow 2$ tilings. Each line of the diagram 4

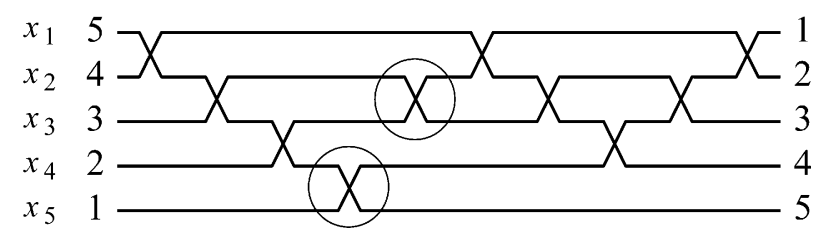

Fig. 4. A diagram associated with a sorting algorithm acting on five element lists. A line follows a number during the sorting process. Each pair of lines cross, only once. Each crossing represents a comparator $[i ; i+1]$. 
represents a de Bruijn line and crosses every other line exactly once. However, different sorting algorithms sometimes represent the same de Bruijn grid, since only the crossing topology is meaningful. For example, in Fig. 4, the fourth and the fifth comparator (i.e. [4;5] and [2;3]) are applied in this order (these comparator are circled in the figure). If they were applied in the reverse order, the algorithm would be different whereas the de Bruijn grid would be the same.

Therefore we define equivalence classes of sorting algorithms. ${ }^{(18,21)}$ We say that two successive comparators $[i ; i+1]$ and $[j ; j+1]$ commute if $|i-j|>1$. Two algorithms are equivalent if they differ by a finite number of comparator commutations. Equivalence classes of $D$-element sorting algorithms are in one-to-one correspondence with $D$-family grids with one line per family, and therefore with tilings inscribed in polygons of side 1. Following Knuth, we denote by $B_{D}$ this number of equivalence classes, whereas the number of algorithms is denoted by $A_{D}\left(A_{D} \geqslant B_{D}\right)$. Since each pattern of crossings (equivalence class of algorithms) defines a tiling, the tiling entropy density per tile is

$$
\bar{\sigma}_{D}=\frac{1}{N_{T}} \log B_{D} .
$$

Sorting algorithms are easy to manipulate and provide efficient enumeration numerical tools. This analogy provides us with the number $B_{D}$ of $D \rightarrow 2$ tilings for small values of $D \leqslant 10$ from the work of $\mathrm{Knuth}^{(18)}$ as extended by us ${ }^{(12)}$ (see Table I). Unfortunately it is not possible to reach the next value $(D=11)$ for the foreseeable future using our current algorithms. Figure 5 suggests that the entropy $\bar{\sigma}_{\infty}(p=1)$ has a well defined limit when $D$ tends to infinity. Indeed, Knuth ${ }^{(18)}$ gives lower and upper bounds on $B_{D}$ from which we get

$$
\frac{1}{3} \log 2 \leqslant \lim _{D \rightarrow \infty} \bar{\sigma}_{D} \leqslant 2 \log 2
$$

Moreover, Björner (see ref. 19, p. 270) derives a better upper bound:

$$
\lim _{D \rightarrow \infty} \bar{\sigma}_{D} \leqslant 1.44 \log 2
$$

In Section 3, we argue that large $D$, fixed boundary tilings, have the same entropy per tile as free boundary ones. Since we demonstrated in paper $\mathbf{I}^{(1)}$ 


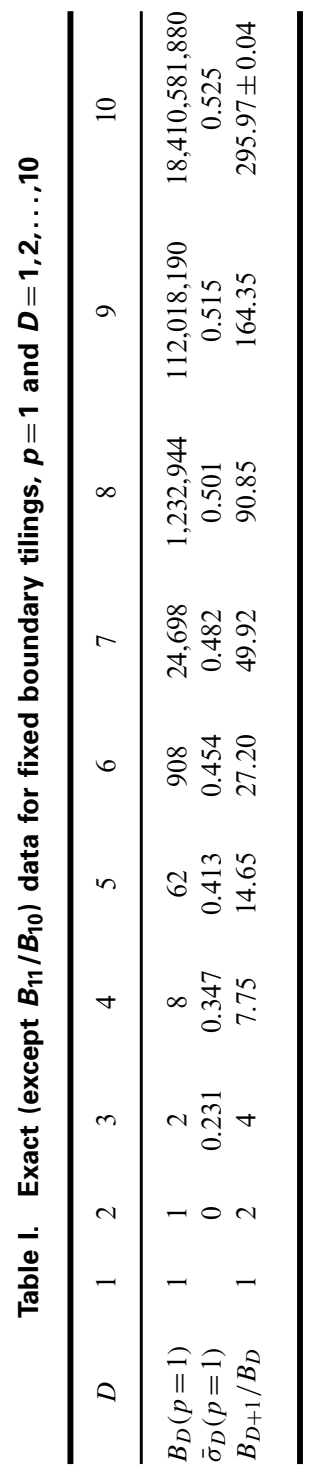




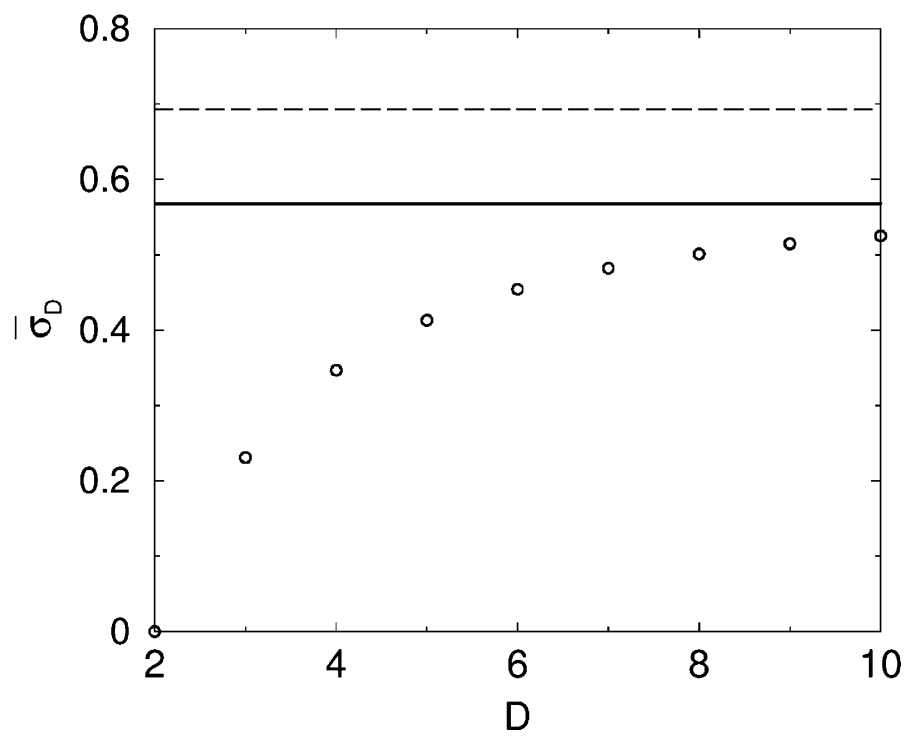

Fig. 5. Entropy per tile $\bar{\sigma}_{D}$ of fixed boundary tilings $(p=1)$. We indicate the limiting values 0.568 (full line) and the close upper bound $\log 2 \simeq 0.693$ (dashed line).

that for free boundary entropies $\lim _{D \rightarrow \infty} \sigma_{D} \leqslant \log 2$, we finally get a better bound:

$$
\lim _{D \rightarrow \infty} \bar{\sigma}_{D} \leqslant \log 2 .
$$

This point of view generalizes to systems with more than one line per de Bruijn family, ${ }^{(15)}$ leading to the definition of partial sorting algorithms for merging pre-ordered lists of numbers. Suppose that we have $D$ families of $k_{i}$ numbers each $(i=1, \ldots, D)$, and that in each family the numbers are presorted in increasing order. Algorithms which order the union of these sets of numbers are called partial sorting algorithms. The ideas are essentially the same as in the previous case, except that, since the numbers of a given family are already ordered, the corresponding lines do not cross within the family. The corresponding diagram is similar to a de Bruijn grid with $D$ families of lines, $k_{i}$ lines in each family. The tilings are equivalence classes of such algorithms. They are inscribed in polygons of sides $k_{1}, \ldots, k_{D}$. In ref. 15 , we derive an analytic expression for $A_{D}\left(k_{1}, \ldots, k_{D}\right)$. However, it proves impossible to calculate $B_{D}$ analytically.

The diagonal case, with $k_{1}=k_{2}=\cdots=k_{D}=p$ will be of special interest below. Let $B_{D}(p)$ denote the number of such tilings. Using the sorting 
Table II. Exact data for fixed boundary tilings, $p=2$ and $D=1,2, \ldots, 6$

\begin{tabular}{ccccccc}
\hline$D$ & 1 & 2 & 3 & 4 & 5 & 6 \\
\hline$B_{D}(p=2)$ & 1 & 1 & 20 & 5,383 & $16,832,230$ & $570,702,721,864$ \\
$\bar{\sigma}_{D}(p=2)$ & & 0 & 0.250 & 0.358 & 0.416 & 0.451 \\
\hline
\end{tabular}

algorithm analogy, we computed the numbers of tilings inscribed in polygons of side $2\left(B_{D}(2)\right)$ for small values of $D$ (see Table II).

\section{THERMODYNAMIC LIMIT}

In this section, we argue that the fixed boundary entropy in the large $D$ limit equals the free boundary entropy and is independent of the phason strain. Proofs are provided in Appendices A and B. Free boundary and fixed boundary entropies can be compared when both are known. For hexagonal tilings with $D=3$, the free and fixed entropies are, respectively, $0.323^{(8)}$ and $0.261 .^{(7)}$ For octagonal tilings with $D=4$ the values are $0.434^{(23)}$ and $0.36(1) .{ }^{(15)}$ Therefore the relative difference $(\sigma-\bar{\sigma}) / \sigma$ decreases between $D=3$ and $D=4$, consistent with a vanishing difference at large $D$. Exact data on $p=1$ and $p=2$ tilings (Tables I and II) suggest that when $D$ becomes large, the entropy becomes independent of $p$.

A glance at Fig. 1 suggests that when $D$ becomes large, the faceted regions of fixed boundary tilings, which are caused by the strong influence of the boundary, ${ }^{(17)}$ occupy a vanishing fraction of the tiling area close to the boundary. In these regions, few families of de Bruijn lines cross, so only a small number of tile types are present. The central region of

the tiling, where a large number of de Bruijn line families cross, becomes dominant as $D$ increases. The coarse-grained entropy density in the central region approaches the large $D$ free boundary entropy $\sigma_{\infty}$.

More precisely, let $\left(x_{i}\right)$ be any set of numbers $0<\alpha \leqslant x_{i} \leqslant \beta$, where $\alpha$ and $\beta$ are fixed positive real numbers. Consider fixed boundary tilings of a $2 D$-gon with edge lengths $k_{i}=x_{i} p$ as $p \rightarrow \infty$. We impose such bounds on the side lengths in order to be sure that our tilings are effectively large codimension ones (see also paper $\mathrm{I}^{(1)}$ (Section 2.3)). The tile fractions are

$$
\bar{n}_{i j}=\frac{k_{i} k_{j}}{N_{T}}=\frac{k_{i} k_{j}}{k_{1} k_{2}+\cdots+k_{D-1} k_{D}},
$$


owing to Eq. (1). In an unstrained tiling, where all $k_{i}$ are equal,

$$
\bar{n}_{i j}^{*}=\frac{2}{D(D-1)} .
$$

These quantities differ noticeably from their free boundary counterparts. ${ }^{(1)}$ The condition $0<\alpha \leqslant x_{i} \leqslant \beta$ imposes

$$
\bar{n}_{i j} \geqslant \frac{\alpha^{2}}{\beta^{2}} \bar{n}_{i j}^{*}=\frac{\alpha^{2}}{\beta^{2}} \frac{2}{D(D-1)} .
$$

This lower bound ensures that no tile fraction is vanishingly small and therefore that the tiling is really a large codimension one rather than a small codimension one with few defect lines added in.

Under this condition, Appendix A proves that the sequence $\bar{\sigma}_{D}$ reaches a thermodynamic limit $\bar{\sigma}_{\infty}$ that does not depend on the set $\left(x_{i}\right)$. Furthermore, these tilings are locally equivalent to free boundary high symmetry tilings. The local entropy density thus approaches $\sigma_{\infty}$, and $\bar{\sigma}_{\infty}=$ $\lim _{D \rightarrow \infty} \bar{\sigma}_{D}=\sigma_{\infty}$. Moreover, this result remains valid if $D$ becomes large while $p$ is held fixed (even in the extreme case $k_{i}=p=1$ ). In particular, the limits $D \rightarrow \infty$ and $p \rightarrow \infty$ commute with each other. The proofs of all these results are given in Appendix B. This insensitivity of entropy to boundaries could be anticipated since we know that phason elastic constants vanish at large $D^{(1)}$ and that, because of the regular character of large $D$ tilings established in Appendix B, the phason gradient $\boldsymbol{E}$ is nearly everywhere bounded.

We find in Appendix A that large $D$ tilings have a central region holding half of the tiles, which contains lines of all de Bruijn families and all tile species and which is nearly homogeneous and strain-free. Away from this central region, the tiling no longer contains lines of all de Bruijn families. We define the effective (coarse-grained) dimension $D_{\text {eff }}$ as the number of de Bruijn families present in a small tiling patch, and the effective codimension $c_{\text {eff }}=D_{\text {eff }}-2$. This effective codimension decreases from $D-2$ at the center to 0 at the boundary. For diagonal tilings $\left(x_{i}=1\right.$ so that $k_{i}=p$ for all $i$ ), where the tiling has a circular symmetry at the large $D$ limit, the effective codimension $c_{\text {eff }}$ varies as

$$
c_{\text {eff }}(r)=(D-2) \gamma(r / R)
$$

where $r$ is the distance to the center and $R$ is the radius of the tiling. The function $\gamma(r / R)$ varies from the value $\gamma(0)=1$ at the tiling center to 
$\gamma(1)=0$ at the boundary. For large $D, c_{\text {eff }}(r)$ diverges for all $r<R$, so the local entropy density approaches $\sigma_{\infty}$ for all $r<R$.

This dependence of $c_{\text {eff }}$ on the location in the tiling can be used to estimate finite size corrections to $\bar{\sigma}_{D}$. Consider a fixed value of $D \gg 2$, and find the number of tiles in the regions where $c_{\text {eff }} \leqslant c_{0}$, with $c_{0} \ll D-2$ some fixed value. These outer regions form an annulus $R_{\mathrm{an}} \leqslant r \leqslant R$. Inside the radius $R_{\text {an }}$, the mean entropy density is nearly $\sigma_{\infty}$, but in the annulus the mean entropy density is $\sigma_{\mathrm{an}}<\bar{\sigma}_{D}$. We can estimate the mean entropy density on the whole tiling of radius $R$ as

$$
\bar{\sigma}_{D} \simeq n \sigma_{\mathrm{an}}+(1-n) \sigma_{\infty},
$$

where $n$ is the fraction of tiles in the annulus.

Now it is shown in Appendix A, Eq. (A.16), that the fraction of tiles in the region of effective codimension $c_{e f f}=x D$ is $n(x)=\psi(x) / D$ where $\psi$ is a regular function tending to $2 / 3$ when $x$ goes to 0 . Hence the fraction of tiles in the annulus

$$
n \simeq \frac{2}{3} \frac{c_{0}}{D}
$$

Finally, we estimate

$$
\bar{\sigma}_{D} \simeq \sigma_{\infty}-\frac{B}{D},
$$

where $B>0$ is a constant related to the entropy difference $\left(\sigma_{\infty}-\sigma_{\text {an }}\right)$ and the chosen value $c_{0}$, both of which are independent of $D$.

This result holds only for a large tiling side length $p$ because the annulus width must be large as compared to a tile edge in order to define properly an entropy per tile. Appendix A.3 says that the effective codimension $c_{\text {eff }}=c_{0}$ at $R_{\text {an }}$ if $R_{\text {an }}=(1-\epsilon) R$ where

$$
\epsilon=\frac{\pi^{2}}{24} \frac{c_{0}^{2}}{(D-2)^{2}} .
$$

The above requirement reads $\epsilon R \gg 1$, that is to say $p \gg D$ with $R=p D / \pi$. The large $p$ limit must be taken before the large $D$ one. Numerical finite $D$ corrections in small $p$ tilings turn out to be of order $1 / D$ but positive (see section 4).

In conclusion, the local structure, and therefore the entropy per tile, of large $D$ tilings is independent of their size, shape, tile fractions and 
boundary conditions. In all these cases, the local structure of these tilings is similar to free boundary large $D$ tilings, where one encounters only one de Bruijn line per family in large tiling patches. ${ }^{(1)}$ In this sense, it is a true thermodynamic limit. These results will be confirmed by numerical simulations in 4.3.

Henceforth, we concentrate on the study of tilings inscribed in $2 D$ gons of sides $k_{i}=p=1$ as representative of the whole class of high symmetry tilings. In particular, we focus on $p=1$ tilings to extract (numerically) the thermodynamic limit of the entropy density.

\section{SIMULATIONS}

Monte Carlo numerical simulations ${ }^{(24)}$ are widespread in the fields of random tilings and quasicrystals where there exist many difficult unsolved theoretical questions. ${ }^{(25)}$ To check our claims of a thermodynamic limit, to obtain $B_{D}$ for $D>10$, and to get more precise numerical values than those obtained via mean-field arguments, ${ }^{(1)}$ we perform Monte Carlo calculations on large $D$ tilings. The $D \rightarrow 2$ configuration space is sampled for large $D$ systems via single-vertex flip dynamics. This method is validated by the connectivity of the space of configurations in two dimensions. ${ }^{(20,21,15)}$

These simulations utilize fixed boundary tilings for the sake of technical convenience. It is easier to code a fixed boundary tiling in the memory of a computer, and the entropy is easier to estimate in this case. We have established that the central region of a large $D$ tiling is close to a free boundary tiling. All the statistics related to free boundary tilings will be collected in such central regions. Note that, as far as unstrained tilings are concerned, we restrict our study to polygonal boundaries of side $p=1$ since we establish that such tilings behave like large $p$ ones when $D$ goes to infinity.

\subsection{Monte Carlo Algorithm}

The algorithm is implemented as follows: at each Monte Carlo step, select a vertex at random, with uniform probability; if this vertex is flippable, then flip it; repeat sufficiently many times. The method is validated by the ergodicity of the space of configurations via flip dynamics: any configuration is reachable from any initial configuration.

A key point is to check that this algorithm samples configurations with uniform probability, since we are only interested in configurational entropy in which all configurations play the same role. Thus we need to establish that this algorithm defines a Markovian process which satisfies the detailed balance condition. ${ }^{(24)}$ 


$$
w\left(\mathcal{C}_{1} \rightarrow \mathcal{C}_{2}\right)=w\left(\mathcal{C}_{2} \rightarrow \mathcal{C}_{1}\right)
$$

where $w\left(\mathcal{C}_{1} \rightarrow \mathcal{C}_{2}\right)$ denotes the transition probability from configuration $\mathcal{C}_{1}$ to configuration $\mathcal{C}_{2}$. When two configurations differ by a single flip, there is only one way to go from one of them to the other one by a single flip. Therefore $w=1 / N_{V}$ where $N_{V}$ is the number of vertices. Since $N_{V}$ is independent of the tiling, relation (14) is established.

Note that the flip acceptance rate equals the fraction (about $31 \%$ as reported in Section 4.3) of vertices with threefold coordination. Vertices with more than threefold coordination cannot be flipped by an "elementary" phason flip. An alternative Monte Carlo dynamics, in which a threefold vertex is flipped in every step, does not obey detailed balance because the number of flippable vertices is not conserved, and hence Eq. (14) is violated.

Another point to consider in Monte Carlo simulations is the correlation time $t_{0}$ (in units of Monte Carlo steps per vertex) which measures how many steps are necessary between samples to avoid excessive sampleto-sample correlations. Even though some recent work brought new results for small $D$ (see ref. 26 and references therein), this question has no rigorous definitive answer for large $D$. However, our numerical estimations of $t_{0}$ are all in agreement with the conjecture $t_{0} \leqslant N_{V} / 2$.

\subsection{Path Counting and Entropy Estimate}

To estimate the entropy $\sigma_{D}$ of fixed boundary tilings, we shall use a path-counting algorithm based upon de Bruijn line enumeration ${ }^{(12)}$ directly derived from the general method described in Section 2.2 of paper $\mathrm{I}^{(1)}$. Figure 6 illustrates the method. At each step, we build a $D+1 \rightarrow 2$ tiling from a $D \rightarrow 2$ one. Starting from any $D \rightarrow 2$ tiling, we choose a path of length $D$ along tile edges, going from bottom to top. We cut the tiling along this path, separating the two parts by length 1 , then draw new bonds connecting previously identical vertices. Finally, we adjust all edge orientations to match the set of symmetry $D+1$.
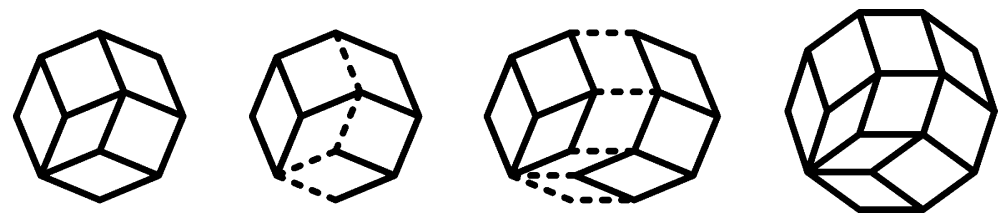

Fig. 6. Iterative construction of fixed boundary tilings $(D \rightarrow 2, p=1)$. 
Let $\bar{P}_{D}$ denote the mean number of bottom-to-top paths on the $D \rightarrow$ 2 tilings:

$$
\bar{P}_{D}=\frac{1}{B_{D}} \sum_{\tau=1}^{B_{D}} P_{D}(\tau),
$$

where $P_{D}(\tau)$ is the exact number of paths on the tiling $\tau$, and $B_{D}$ is the number of such tilings. Then

$$
B_{D+1}=\bar{P}_{D} B_{D}
$$

Iterating relation (16), and taking the logarithm yields

$$
\log B_{D}=\sum_{k=2}^{D-1} \log \bar{P}_{k} .
$$

For large $D$, the ratio $\frac{1}{D} \log \bar{P}_{D}$ approaches a finite limiting value. Taking the limit of (17) as $D \rightarrow \infty$, and noting Eqs. (1) and (2), we find

$$
\lim _{D \rightarrow \infty} \bar{\sigma}_{D}=\lim _{D \rightarrow \infty} \frac{1}{D} \log \bar{P}_{D}
$$

Hence, by accumulating statistics on the number of paths allowed on tilings, we may evaluate the entropy.

We use Monte Carlo sampling to generate an ensemble of $D \rightarrow 2$ tilings. On each tiling $\tau$ we can quickly evaluate $P_{D}(\tau)$ using a generalization of the Pascal Triangle construction: starting at the bottom of the tiling, assign each vertex an integer value equal to the number of paths that reach it from the bottom. The value at any vertex is iteratively the sum of the values at each prior vertex to which it is connected (see Fig. 7). At the end of the process, the value of the top vertex is $P_{D}(\tau)$.

We tested this algorithm on small $D$ systems, where numerical values are in good agreement with the exactly known mean number of paths $\bar{P}_{D}=$ $B_{D+1} / B_{D}$ (Table I). For example, Table III shows the convergence towards the exact value for $D=9$ tilings. For large $D$ systems, simulations are our only means of obtaining information about path count statistics, which are displayed in Fig. 8. In this figure, error bars are calculated as follows: the standard deviation $\Delta$ is measured numerically. The error is estimated by $\Delta / \sqrt{I}$, where $I$ is the number of independent measures in the simulation (that is ${ }^{(24)}$ its total length divided by twice the correlation time $t_{0}$ ). Note 


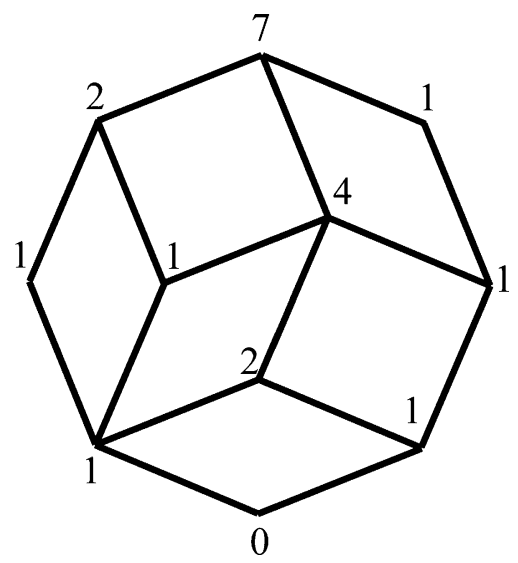

Fig. 7. Path count construction, as described in the text.

Table III. Convergence of $D=9$ path count data for increasing run length. Measured numerical errors on the latter quantity (Num. err.) are always smaller than estimated error bars (Estim. err.). The latter are calculated using numerically measured standard deviations and conjectured auto-correlation times (Section 4.1 ${ }^{(24)}$ )

\begin{tabular}{|c|c|c|c|c|c|c|}
\hline$N_{M C}$ & $10^{3}$ & $10^{4}$ & $10^{5}$ & $10^{6}$ & $10^{7}$ & exact \\
\hline $\bar{P}_{9}$ & 159.264 & 164.176 & 164.411 & 164.371 & 164.344 & \\
\hline $\log \bar{P}_{9} / 9$ & 0.563396 & 0.566771 & 0.566930 & 0.566903 & 0.566884 & 0.566891 \\
\hline Num. err. & $3.510^{-3}$ & $1.210^{-4}$ & $3.910^{-5}$ & $1.210^{-5}$ & $610^{-6}$ & \\
\hline Estim. err. & $8.510^{-3}$ & $2.710^{-3}$ & $8.510^{-4}$ & $2.710^{-4}$ & $8.510^{-5}$ & \\
\hline
\end{tabular}

that the actual errors calculated in Table III are compatible with the previous error bar.

This graph presents a maximum near $D=20$ which is related to finite $D$ and finite size corrections. Paper $\mathrm{I}^{(1)}$ shows that for free boundary systems, finite $D$ corrections to $\log \bar{P}_{D} / D$ are of order $1 / D^{2}$. Section 3 of the present paper argues for negative $1 / D$ finite size corrections with fixed boundary conditions in the large $p$ limit. Here, though, we examine $p=1$ systems with one de Bruijn line per family. Our numerical results indicate that corrections also fall off as $1 / D$ to the first power. Combining these corrections suggests

$$
\frac{\log \bar{P}_{D}}{D}=\bar{\sigma}_{\infty}+\frac{A}{D}-\frac{B}{D^{2}}+O\left(\frac{1}{D^{3}}\right)
$$




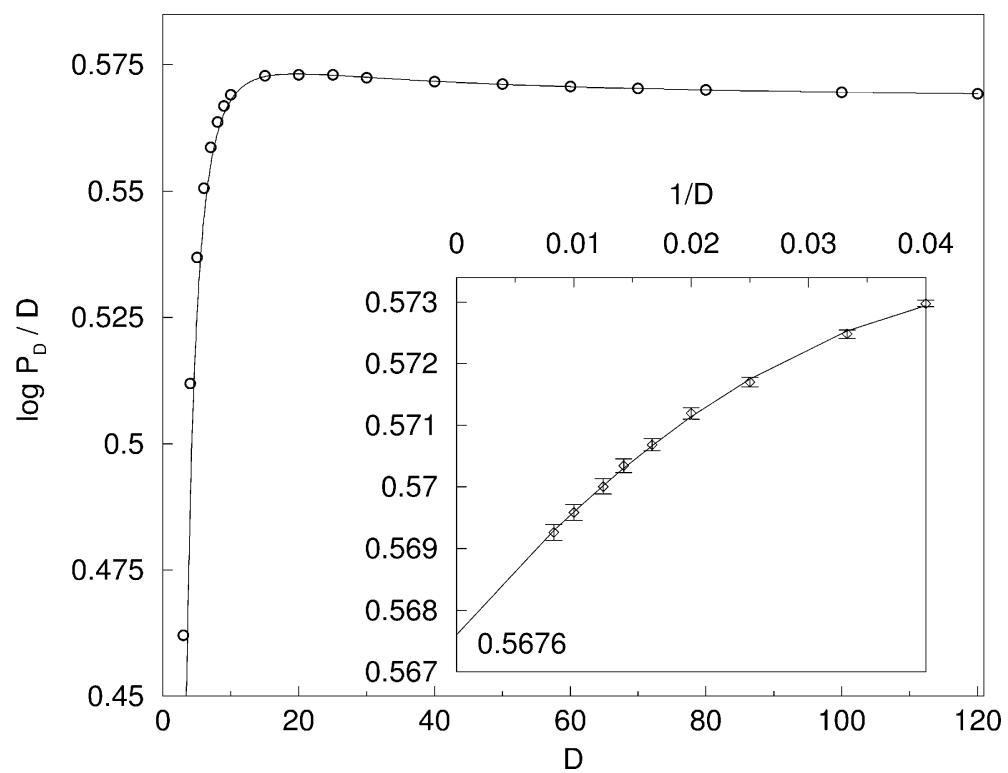

Fig. 8. Value of $\log \bar{P}_{D} / D$ as a function of $D$ up to $D=120$; inset: large $D$ behavior as a function of $1 / D$ and quadratic fit. The numerical values (symbols) as well as the fitted ones (lines) present a maximum near $D=20$.

The numerical results in Fig. 8 reproduce this behavior. All numerical data beyond $D=15$ coincide with the fitted ones up to error bars. In particular, the maximum observed near $D=20$ is reproduced. We estimate the limiting value of $\log \bar{P}_{D} / D=0.5676 \pm 0.0001$. The uncertainty estimate on this last limit comes from excluding the data with $D=120$. These observations provide the first order correction to the entropy at $p=1$ : relation (17) reads

$$
\log B_{D}(1) \simeq \sum_{k=2}^{D-1} k\left(\sigma_{\infty}+\frac{A}{k}\right) \simeq \frac{D(D-1)}{2} \sigma_{\infty}+A(D-2)
$$

and

$$
\bar{\sigma}_{D}(p=1)=\frac{2}{D(D-1)} \log B_{D}(1) \simeq \sigma_{\infty}+\frac{2 A}{D} .
$$

Note that our simulations yield $A>0$ which means that these corrections of order $1 / D$ are positive. By contrast, corrections to $\sigma_{\infty}$ in relation 
(12) are also of order $1 / D$ but negative. The sign of these large $p$ corrections is the combination of two effects: the paths visit low codimension regions near the boundary, which should increase the entropy (e.g., a path running on a square grid of codimension 0 has an entropy density of $\log 2>\sigma_{\infty}$ ); the entropic repulsion between paths near the top and bottom vertices, where they are crowded, decreases the entropy. In the $p=1$ case, paths are not subject to entropic repulsion. Presumably the coefficient $A$ in Eq. (19) is a function of $p$ and changes its sign when $p$ grows.

\subsection{Thermodynamic Limit Revisited}

Here we test the results of Section 3 where it was demonstrated that the local structure and the entropy per tile of tilings becomes independent of boundary conditions when $D$ becomes large. We compare relevant numerical quantities both in the whole tiling and in the central region, that is supposed to be close to a free boundary tiling. We concentrate on vertex coordination numbers which are indicators of the local structure and on path counting which is related to entropy. In Section 4.3.3, we focus on strained fixed boundary tilings.

\subsubsection{Vertex Statistics}

Other pertinent information available from Monte Carlo simulations concerns vertex statistics. They are indicators of the tiling's local "microscopic" structure. They also are a fundamental ingredient of the mean-field approach of paper $\mathrm{I}^{(1)}$ We focus on quantities which can be computed in the mean-field theory, especially coordination numbers and related quantities. We gather statistics both in the whole tiling (excluding vertices actually on the boundaries) and in a central region containing about $20 \%$ of the vertices. Presumably, the central region is only weakly influenced by the fixed boundaries, and we take that data as representative of free boundary tilings.

We have run Monte Carlo simulations up to $D=100$. After plotting the data as functions of $1 / D$, we extrapolate the limiting values via quadratic fits. Figure 9 illustrates two examples of coordination number statistics. We find the fractions of vertices (in the whole tiling and in the central region) whose coordination numbers $Z=3$ and the fractions whose coordination numbers $Z=5$. For finite $D$ we see the central region has a relatively small fraction of tiles with $Z=3$ in comparison with the whole tiling, and a relatively large fraction with $Z=5$. The boundary regions are thus more likely to have $Z=3$ and less likely to have $Z=5$ than a free boundary tiling. However, the extrapolated values for $D \rightarrow \infty$ agree 

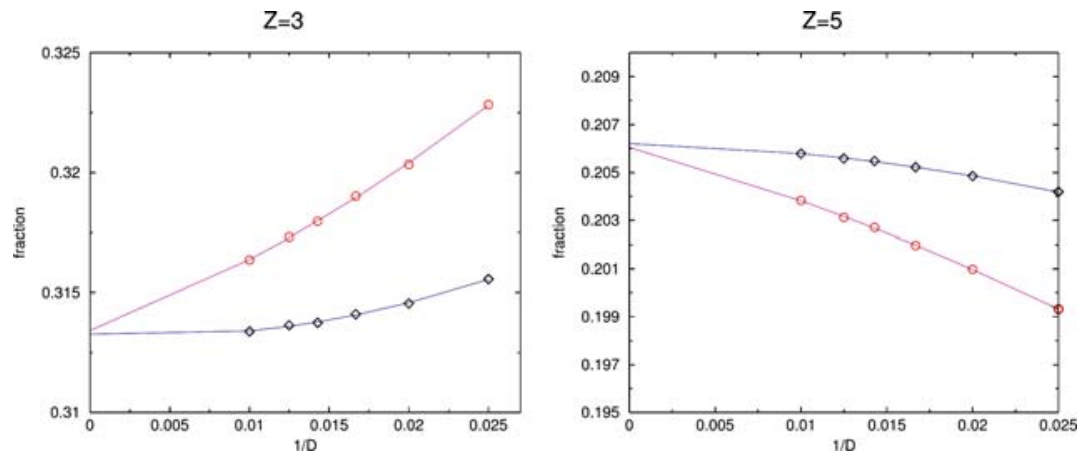

Fig. 9. Fractions of $Z=3$ and $Z=5$ vertices in $D \rightarrow 2$ vertices as a function of $1 / D$, and quadratic fits. Diamonds concern the central region $(20 \%$ vertices), whereas circles concern the whole tiling. The extrapolated large $D$ values coincide.

to within the accuracy of the extrapolation. The same conclusion holds for $Z=4$ (not represented here). For larger values of $Z$, the fractions of vertices are too small and we did not obtain relevant measures. Recall that the mean value of $Z$ is exactly four in an infinite tiling, according to Euler's theorem.

Hence, in the limit $D \rightarrow \infty$ the whole tiling exhibits coordination statistics similar to a free boundary tiling. This point supports the existence of a thermodynamic limit - the local structure becomes uniform throughout the tiling, independent of proximity to the boundaries and the local strain they create.

Other quantities we examined relate directly to mean-field theory and were reported in paper $\mathrm{I}^{(1)}$. Our mean-field theory was based upon the number of choices to be made while inscribing directed paths on tilings. The number of choices $N_{c}(v)$ at a vertex $v$ is the number $p$ of "arms" emerging from a vertex. The number $q$ of "legs" leading into a vertex together with the number of arms obey $p+q=Z$. Paper I reports the simulated probability distribution for $p$ and mean value of $p q$, and shows these agree well with mean-field theory. Here, we display in Table IV the probability distribution for $Z$, and compare it with the predictions of mean-field theory. The agreement is also satisfactory.

An intriguing feature of the fixed boundary tilings is the divergence of tile vertex density near the boundaries,

$$
d(r / R) \simeq \frac{1}{\sqrt{6(1-r / R)}}
$$


Table IV. The first values of the limiting distribution of coordination numbers $Z$, obtained both in the mean-field approximation and numerically, by Monte Carlo simulations

\begin{tabular}{cccccc}
\hline$Z$ & 3 & 4 & 5 & 6 & 7 \\
\hline Mean-field & 0.33 & 0.41 & 0.20 & 0.05 & 0.009 \\
Numerical & 0.31 & 0.43 & 0.21 & 0.04 & 0.005 \\
\hline
\end{tabular}

caused by the vanishing of tile area, and established in Appendix (A.3) for a particular tiling. This effect is plainly visible in Fig. 1. Given this spatial nonuniformity it is natural to worry about the path counting arguments because the choice statistics might vary among different portions of the whole tiling. It turns out this does not happen. Figure 10 illustrates this point by plotting vertex statistics averaged over different portions of the tiling. We divided tilings into 20 concentric circular bins and evaluated in each bin the average vertex density and the average fractions of vertex with each coordination number $Z$. The results are illustrated for the cases $D=60,92,120$. Clearly the vertex density converges to a non-constant function which diverges at the tiling boundary according to the prediction of Eq. (22). However, the fractions of each vertex type rapidly

\section{Radial Variation}

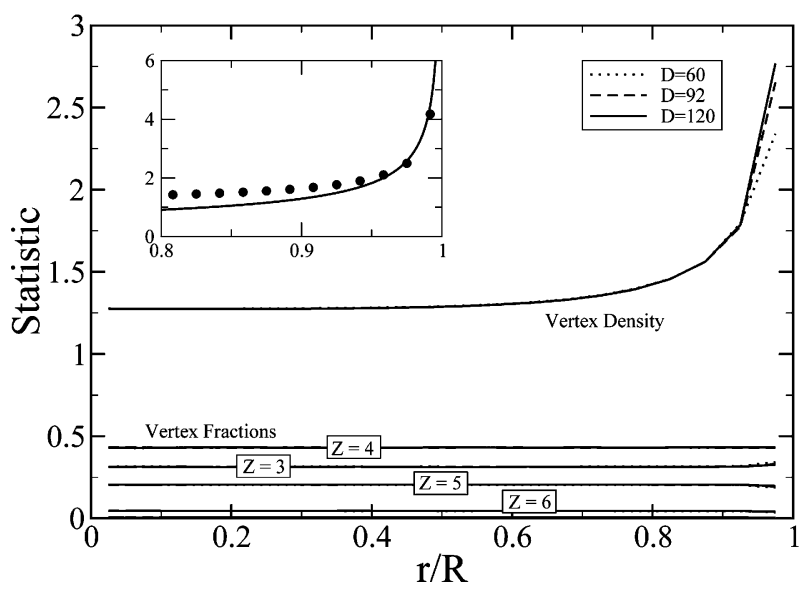

Fig. 10. Radial variation of vertex statistics from tiling center to edge. Only the vertex density shows spatial variation. Inset: vertex density for $D=200$ (points) matches the function $1 / \sqrt{6(1-r / R)}$ (line) as $r / R \rightarrow 1$. 
converge to their large $D$ limit independent of position within the tiling. Consequently the entropy per vertex should be uniform, even though the entropy per area diverges due to the diverging vertex density.

\subsubsection{Path Counting in the Central Region}

To confirm that the entropy per tile (or vertex) is independent of boundary conditions, we repeat calculations like Section 4.2, but we concentrate on the central region. It is reasonable to suppose that statistics in the central region should match the statistics inside free boundary tilings.

On a $D \rightarrow 2$ tiling we define $D+1$ layers of vertices as follows: layer 0 contains only the bottommost vertex $v_{0}$, layer 1 contains the vertices at distance 1 from $v_{0}$, layer $k$ contains the vertices at distance $k$ from $v_{0}$ when one follows bottom-to-top paths. Layer $D$ contains only the topmost vertex $v_{D}$. Let $\bar{P}_{D}^{\prime}$ denote the mean number of length $D / 2$ paths from a given vertex $v_{\text {start }}$ of layer $D / 4$ to any vertex of layer $3 D / 4$. For convenience, we take $D$ a multiple of 4 , and also require that $v_{\text {start }}$ is close to the vertical diameter (see Fig. 11). Most paths stay close to this central diameter because their typical deviation from this diameter grows like $\sqrt{D}$ whereas the size of the tiling grows like $D$. Consequently, $\bar{P}_{D}^{\prime}$ counts paths in the central region. Data is averaged over many independent tilings.

Fitting finite $D$ data as in Section 4.2, we find the limiting value

$$
\lim _{D \rightarrow \infty} \frac{\log \bar{P}_{D}^{\prime}}{D / 2}=0.5670 \pm 0.0005 .
$$

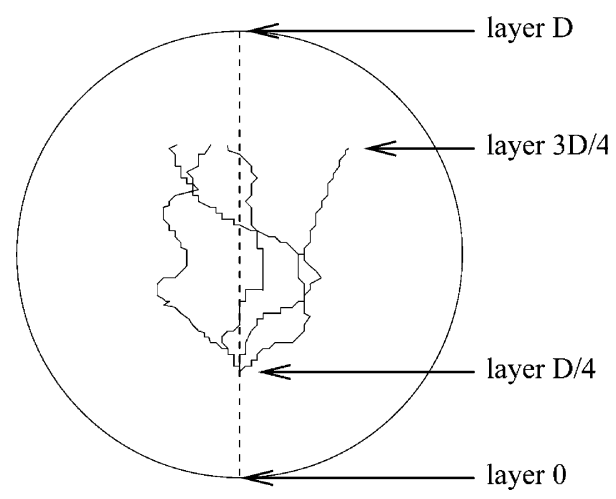

Fig. 11. Paths in the central region going from a vertex in layer $D / 4$ close to the vertical diameter to any vertex in layer $3 D / 4$. 
This value coincides with its fixed boundary counterpart up to error bars. If the path counting statistics in large $D$ fixed boundary tilings were influenced by boundary conditions, we would have expected the central value to differ from the value over the entire tiling. We conclude that the fixed boundary entropy per tile matches the free boundary entropy in the $D \rightarrow \infty$ limit.

\subsubsection{Phason Strained Tilings}

The data just reported in Section 4.3.1 suggests that the tiling structure does not depend on the phason strain, since statistics are identical in the central region and in the boundary vicinity. We want to test this point directly by performing simulations on phason strained fixed boundary tilings.

We consider three different forms of phason strain in our numerical simulations: low frequency strain in which $D / 2$ consecutive line families occur with $k_{i}=1(i=1, \ldots, D / 2)$, the remaining $D / 2$ consecutive line families occur with $k_{i}=2(i=D / 2+1, \ldots, D)$; the same low frequency with a larger amplitude so that $k_{i}=3(i=D / 2+1, \ldots, D)$ replaces $k_{i}=2$; high frequency strain with $k_{i}=1$ ( $i$ odd) and $k_{i}=2$ ( $i$ even). Figure 12 illustrates an equilibrated tiling with applied phason strain. The tiling has $D=30$ and the strain is low frequency and large amplitude.

Computer simulations of path count statistics for $D=10, \ldots, 50$ reveal no significant dependence of path count on phason strain amplitude

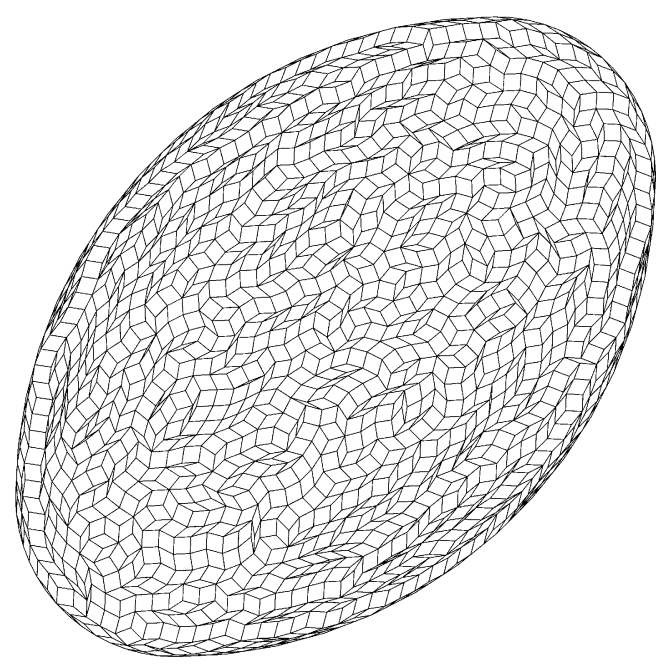

Fig. 12. Phason strained large $D$ tiling. The added paths still go from bottom to top. 
Table V. Path count statistics for strained tilings

\begin{tabular}{ccccc}
\hline$D$ & $p=1,1, \ldots$ & $p=1,1, \ldots, 2,2, \ldots$ & $p=1,1, \ldots, 3,3, \ldots$ & $p=1,2,1,2, \ldots$ \\
\hline 10 & 0.569 & 0.572 & 0.572 & 0.573 \\
20 & 0.573 & 0.572 & 0.571 & 0.574 \\
30 & 0.572 & 0.571 & 0.570 & 0.572 \\
40 & 0.572 & 0.570 & 0.570 & 0.571 \\
50 & 0.571 & 0.571 & 0.570 & 0.570 \\
\hline
\end{tabular}
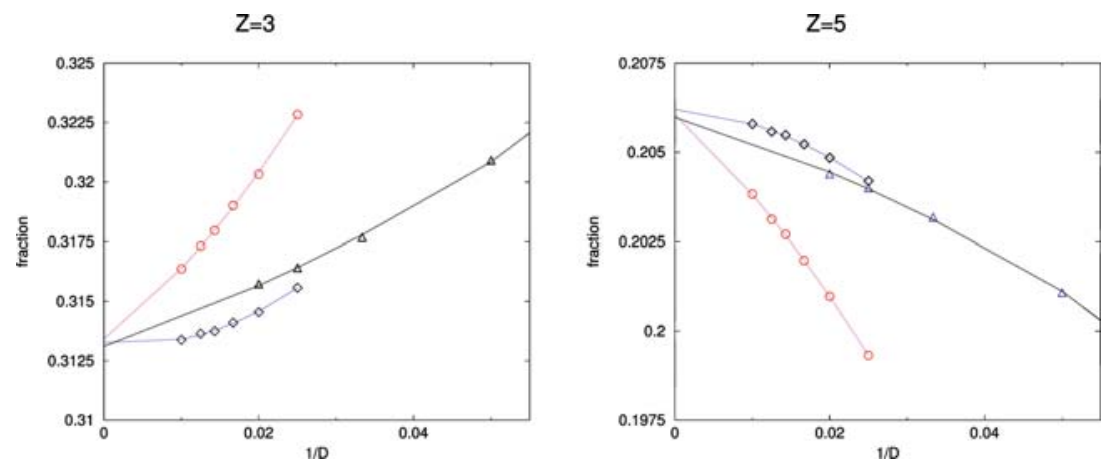

Fig. 13. Fractions of $Z=3$ and $Z=5$ vertices in strained tilings $(p=1,1, \ldots, 3,3, \ldots)$ (triangles), together with previous data on fixed boundary unstrained tilings (circles) and free boundary ones (diamonds). The limiting values coincide with the previous one, up to statistical errors. Lines are quadratic fits.

or frequency. The small variations seen (see Table V) cannot be separated from finite size effects. Thus entropy does not depend on phason strain, supporting a posteriori arguments developed for free boundary tilings. ${ }^{(1)}$ Recall that strain-independence of entropy in fixed boundary tilings relies upon strain-independence in free boundary tilings (see Section 3).

The same conclusion holds concerning vertex statistics on coordination number and choice distributions. No significant differences are found in comparison to unstrained fixed boundary tilings or free boundary tilings (see Fig. 13). These data support the existence of a universal local structure, independent of strain and boundary conditions in the limit $D \rightarrow \infty$.

\section{CONCLUSION}

This paper tackles random tilings of high symmetry with fixed boundary conditions. In random tiling theory, boundary conditions are crucial because finite codimension fixed boundary tilings have a lower entropy 
than free boundary ones. However, we argue that boundaries become irrelevant when the codimension becomes large. We also demonstrate that $p=1$ tilings with one de Bruijn line per family have the same entropy per tile as tilings with $p$ large and even infinite, for sufficiently large $D$.

As a consequence, the numerical study of the entropy of large codimension random tilings can be concentrated on fixed boundary tilings filling a $2 D$-gon of side lengths set to 1 . We perform exact enumeration for $D \leqslant 10$, thanks to an analogy between fixed boundary tilings and some class of sorting algorithms. We use Monte Carlo simulations for larger tilings, up to $D=120$. In both cases, the fact that tilings have a fixed polygonal boundary greatly simplifies their encoding and their manipulation in the memory of a computer. Our Monte Carlo data analysis is based on the same iterative process (i.e. concentrating on values of $\bar{P}_{D}$ ) as our mean-field theory in paper $\mathrm{I}^{(1)}$ We obtain a very accurate estimate of the entropy per tile, $\bar{\sigma}_{\infty}=0.5676 \pm 0.0001$, compared to the approximate mean-field value $\sigma_{\infty}^{M F} \simeq 0.598$.

Insensitivity of the entropy per tile and vertex statistics to boundary conditions at large $D$ restores a thermodynamic limit which does not exist at finite $D$. However, caution is required. Even if "topological" quantities such as entropy and vertex statistics become homogeneous in the tiling, its "metric" properties remain heterogeneous, as discussed in Section 4.3.1. Consequently we find the entropy per vertex is homogeneous while the entropy density per area diverges near the tiling boundary.

Many of our methods and results can be generalized to higher dimensional tilings. However, the generalization may not be simple to implement in practice. Indeed the de Bruijn directed paths become directed surfaces built of faces of rhombohedra. The description of these surfaces in terms of successive choices must be generalized, which highly complicates their enumeration.

The restoration of the thermodynamic limit in the high symmetry limit should remain valid in three- or higher-dimensions, because the vision of large regions with at most one de Bruijn surface per family still holds. The explicit proof should be similar to the Appendix A, with only minor changes to account for the possible spontaneous decomposition into regions of lower effective codimension (as in the "arctic octahedron", see ref. 14). Presumably, the effective codimension still goes to infinity for almost all tiles.

One of the main motivations for the study of high codimension random tilings was the hope that mean-field theory might become exact in the high codimension limit. The principal conclusion of this numerical study, together with the mean-field results of paper I, is that the mean-field theory does not become exact, at least in the simplest version which neglects 
vertex correlations. Rather, the high codimension limit is quite nontrivial, and an exact analytic solution is yet to be found.

\section{APPENDIX A: THERMODYNAMIC LIMIT OF TWO-DIMENSIONAL FIXED BOUNDARY TILINGS}

In this technical appendix, we demonstrate that the fixed boundary entropy $\bar{\sigma}_{D}$ attains a finite limit $\bar{\sigma}_{\infty}$ when $D \rightarrow \infty$, and that this limit coincides with the free boundary entropy $\sigma_{\infty}$. Moreover, we argue that this limit is shared with finite side polygonal boundaries as well as non-diagonal tilings.

Our demonstration relies on the variational principle introduced in refs. 10, 11 to characterize the entropy gradient between the center and the boundary in typical tilings with given fixed boundaries. Typical tilings are those which maximize an entropy functional defined as the integral over the tiling of a local entropy per tile; This local entropy is the free-boundary entropy calculated with the local fractions of tiles. The entropy per tile of fixed boundary tilings is the maximum of this functional.

We show below that, given any domain in the tiling, when $D$ goes to infinity, the tiling in this domain is a piece of (free) $D^{\prime} \rightarrow 2$ tiling, such that $D^{\prime}$ goes to infinity as $D$ does. Hence the local entropy per tile tends to the large $D$ free boundary entropy $\sigma_{\infty}$ (nearly) everywhere and fixed boundary tilings have the same entropy as free boundary ones.

We first consider the case where the sides of the polygonal boundary share the same length $p$ (diagonal tilings), and take the large $p$ limit before taking the large $D$ limit. Second, we consider the case were $D$ becomes large at fixed $p$. We also discuss the non-diagonal case where these side lengths might be different. Our presentation is heuristic but can be made rigorous. Finally, we examine scaling laws for several quantities studied in the paper, such as the effective codimension, when they are written as functions of the radius $r$ from the tiling center.

\section{A.1. Diagonal Tilings}

Our demonstration uses a particular tiling denoted as $\mathcal{T}_{0}$. It is the dual of a particular de Bruijn grid,,$^{(1,3)}$ consisting of $D$ families of $p$ straight lines equally spaced out and forming a regular fan ${ }^{(27)}$ (see Fig. 14, left). The de Bruijn families are denoted by $F_{1}, F_{2}, \ldots, F_{D}$, and are labeled counterclockwise so $F_{k}$ makes an angle $k \pi / D$ with an arbitrary reference direction. Figure 14 displays such a grid and its dual tiling. For finite $D$, such a tiling is known ${ }^{(11)}$ not to maximize the entropy functional among the tilings with the same boundary (see below). By construction, the tiling 
$\mathcal{T}_{0}$ is divided into domains where only a fraction of the de Bruijn families intersect. In each domain, the tiling is homogeneous, with a well defined local entropy per tile at large $p$. Since only $D^{\prime}<D$ de Bruijn families intersect in such a domain, this entropy a priori differs from the free boundary $D \rightarrow 2$ one. However, by rotational symmetry, all the domains where exactly $D^{\prime}$ families intersect have the same local entropy. In the following, the union of such domains where only $D^{\prime}$ families meet will be denoted by $\mathcal{A}_{D^{\prime}}$ and called the region of "effective dimensionality" $D^{\prime}$.

Define $S_{0}^{D \rightarrow 2}$ as the entropy functional evaluated at $\mathcal{T}_{0}$. It satisfies $S_{0}^{D \rightarrow 2} \leqslant \bar{\sigma}_{D}$. Then

$$
\lim _{D \rightarrow \infty} S_{0}^{D \rightarrow 2} \leqslant \bar{\sigma}_{\infty} \leqslant \sigma_{\infty}
$$

Next we intend to show that for any real number $h<1$,

$$
\lim _{D \rightarrow \infty} S_{0}^{D \rightarrow 2} \geqslant h \sigma_{\infty}
$$

It then follows that

$$
\lim _{D \rightarrow \infty} S_{0}^{D \rightarrow 2} \geqslant \sigma_{\infty}
$$
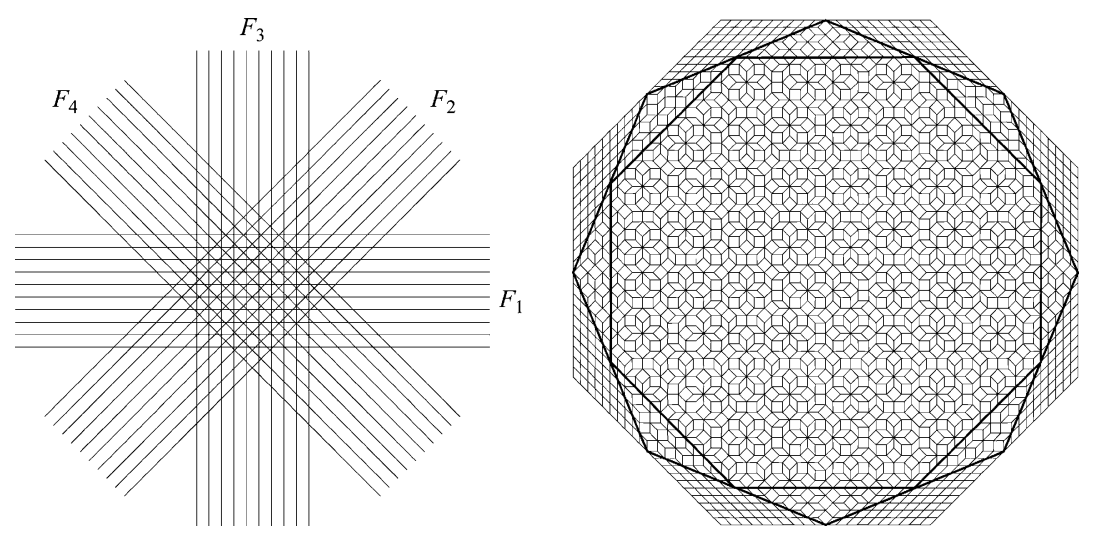

Fig. 14. The de Bruijn straight lines (left) and the corresponding rhombus tiling in the $4 \rightarrow$ 2 case with $p=20$ (for clarity's sake, we have only drawn 10 lines per family instead of 20 on the left figure). The regions where only three or even two families intersect clearly appear on the left figure. The corresponding regions are delimited by the two internal octagons on the right figure: in the outer region $\mathcal{A}_{2}$, only two families meet; In the intermediate one $\mathcal{A}_{3}$, three families meet; And in the central region $\mathcal{A}_{4}$, all four families intersect. 

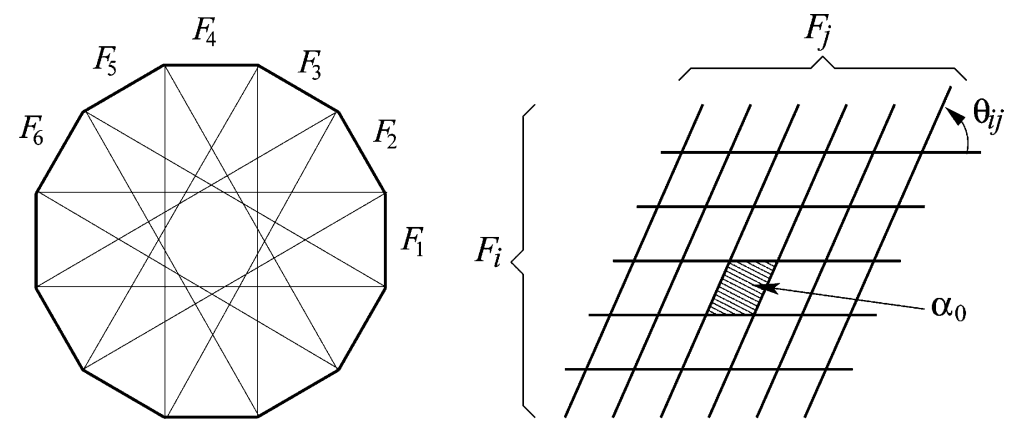

Fig. 15. Left: Schematical representations of the $D$ families of de Bruijn lines and of their intersections, to be compared to Fig. 14 (left). When $D$ tends to infinity, the polygonal boundary tends toward a circle of circumference $2 D p$ and the polygonal central region $\mathcal{A}_{D}$ tends toward a circle a diameter $p$. Right: Two families of de Bruijn lines making an angle $\theta_{i j}$. The number of intersections of these two families in a region equals its area $\alpha$ divided by the area $\alpha_{0}$ of the grayed unit cell.

and, owing to relation (A.1), that

$$
\sigma_{\infty} \leqslant \lim _{D \rightarrow \infty} S_{0}^{D \rightarrow 2} \leqslant \bar{\sigma}_{\infty} \leqslant \sigma_{\infty}
$$

and therefore that the three involved quantities are equal.

Let us prove the above statement (A.2). We use the fact (see Section 4.3.3 and also paper $\mathrm{I}^{(1)}$ ), that the entropy $\sigma_{\infty}$ of free boundary tilings does not depend on strain in the large $D$ limit. We show that the local entropy of $\mathcal{T}_{0}$ equals $\sigma_{\infty}$ in (nearly) all regions $\mathcal{A}_{D^{\prime}}$.

First we estimate how many tiles each region contains. Since a tile is defined as the intersection of two de Bruijn lines, we will count the number of such intersections in a given region, by calculating the number of intersections per unit area in each region on the one hand and the area of the regions on the other hand. However we work not in the "tiling metric" but in the "grid metric" instead. That is to say, in the figure where the de Bruijn lines were originally drawn straight (left-hand representation in Fig. 14). Figure 15 (left) displays a schematic representation of a grid were the different regions under consideration clearly appear.

The number of intersections in a region $\mathcal{A}$ of area $\alpha$ is the sum over all pairs of de Bruijn families of the number of intersections of a pair. Consider a given pair of families $F_{i}$ and $F_{j}, i<j$, which make an angle $\theta_{i j}$. The number of such intersections is the area $\alpha$ divided by the area $\alpha_{0}$ of the unit cell of the lattice defined by these (only) two families (Fig. 15, right). If the distance which separates two lines of a family is set to 1 , then $\alpha_{0}=1 /\left|\sin \theta_{i j}\right|$. Hence the total number of intersections is 


$$
\sum_{i<j} \frac{\alpha}{1 /\left|\sin \theta_{i j}\right|}=\alpha \sum_{i<j}\left|\sin \theta_{i j}\right|
$$

where the indices $i$ and $j$ run over the families present in $\mathcal{A}$.

As displayed in Fig. 15 (left) a region $\mathcal{A}_{D^{\prime}}$ is a crown made of $2 D$ kites (or triangles for the inner crown) of equal areas. A kite (or triangle) of $\mathcal{A}_{D^{\prime}}$ will be denoted by $\mathcal{K}_{D^{\prime}}$ and its area by $\kappa_{D^{\prime}}$. Henceforth the area of $\mathcal{A}_{D^{\prime}}$ is equal to $\alpha_{D^{\prime}}=2 D \kappa_{D^{\prime}}$. In $\mathcal{K}_{D^{\prime}}$, there are $D^{\prime}$ adjacent families of lines, for example $F_{1}, \ldots, F_{D^{\prime}}$. The angles that they make relatively to an arbitrary reference direction are therefore $\theta_{l}=\theta_{0}+l \pi / D, l=1, \ldots, D^{\prime}$ and the number of intersections in $\mathcal{K}_{D^{\prime}}$ is

$$
Q_{D^{\prime}}=\kappa_{D^{\prime}}\left(\sum_{1 \leqslant i<j \leqslant D^{\prime}} \sin \frac{(j-i) \pi}{D}\right) .
$$

When $D$ goes to infinity, $x=D^{\prime} / D$ becomes a continuous variable which represents a "fraction" of the $D$ families of lines. For example, the previous sum in Eq. (A.6) can be estimated by an integral on the variables $y=j / D$ and $z=i / D$. If $E(x)$ denotes the integral part of $x$, then

$$
\begin{aligned}
\phi_{D}(x) & \equiv \sum_{1 \leqslant i<j \leqslant E(x D)} \sin \frac{(j-i) \pi}{D} \simeq D^{2} \int_{0}^{x} d y \int_{0}^{y} d z \sin \pi(y-z) \\
& =\frac{D^{2}}{\pi^{2}}[\pi x-\sin \pi x] .
\end{aligned}
$$

The central region $\mathcal{A}_{D}$ plays a particular role among the regions $\mathcal{A}_{D^{\prime}}$ : when $D$ is large, the fraction of tiles in $\mathcal{A}_{D}$ tends to $1 / 2$. Indeed, as illustrated in Fig. 14 (left) and 15 (left), the central region tends toward a circle of diameter $p$. Its area is $\pi p^{2} / 4$, and, owing to the above result applied to the case $x=1$, the number of intersections that it contains grows like

$$
\frac{D^{2}}{\pi} \frac{\pi p^{2}}{4}=\frac{D^{2} p^{2}}{4} .
$$

Since the total number of tiles grows like $\frac{D^{2} p^{2}}{2}$, this central region contains one half of the total number of tiles.

By comparison, the situation is completely different in the other regions $A_{D^{\prime}}$, with $D^{\prime}<D$. In this case, when $D$ goes to infinity, the fraction of tiles in such a region vanishes. Let us first explicitly compute the 

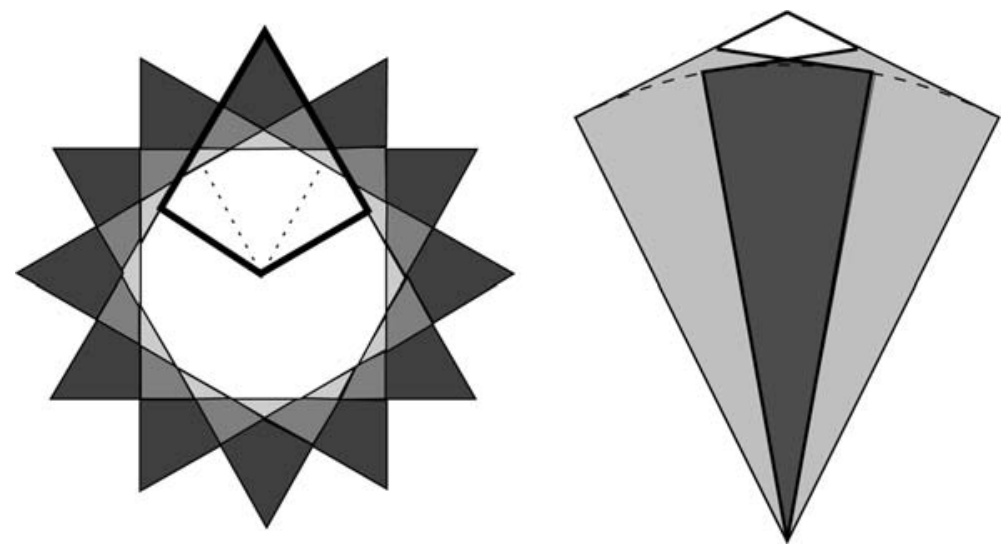

Fig. 16. (left) around the central region $\mathcal{A}_{D}$, there is a first crown of triangles $\mathcal{C}_{D-1}$ (light gray), a second crown of kites $\mathcal{K}_{D-2}$ (medium gray), a third crown of kites $\mathcal{K}_{D-3}$ (dark gray) and so forth; in this figure, $D=6$. Right: the three kinds of kites which define a kite-shaped region $\mathcal{K}_{D^{\prime}}$ (white): a big kite minus two small kites (light gray) plus a smaller kite (dark gray).

asymptotic behavior of this fraction and then discuss how to handle this vanishing character.

Figure 16 (left) shows the geometry of the regions under consideration when $D$ is finite. Around the central region $\mathcal{A}_{D}$, there is a first crown triangular domains, then a second crown of kite-shaped regions, and as $k$ increases, the regions $\mathcal{A}_{D^{\prime}}$ lie in concentric crowns around the central one. Each crown contains $2 D$ kite-shaped (or triangle-shaped) regions. As Fig. 16 (right) illustrates, such a kite can be seen as a greater rectangular kite ${ }^{(28)}$ minus two equal rectangular kites. These two latter kites have a non empty intersection, which is itself an even smaller kite. If the areas of these kites are, respectively, denoted by $\delta_{D^{\prime}}, \delta_{D^{\prime}+1}$ and $\delta_{D^{\prime}+2}$, then the area of $\mathcal{K}_{D^{\prime}}$ is

$$
\kappa_{D^{\prime}}=\delta_{D^{\prime}}-2 \delta_{D^{\prime}+1}+\delta_{D^{\prime}+2}
$$

Now, for each of these four kites, its edges are two rays of the circular central region and two tangents to this central region, perpendicular to these two latter rays. Since the central region has diameter $p$, if the two rays make an angle $\theta$, the area of the kite is

$$
\delta(\theta)=\frac{p^{2}}{4} \tan \left(\frac{\theta}{2}\right)
$$


For the domain $\mathcal{K}_{D^{\prime}}, \theta=\pi\left(D-D^{\prime}\right) / D$ for the greater kite. Henceforth,

$$
\delta_{D^{\prime}}=\frac{p^{2}}{4} \tan \left(\pi \frac{D-D^{\prime}}{2 D}\right) .
$$

The same formula holds for $\delta_{D^{\prime}+1}$ and $\delta_{D^{\prime}+2}$. Thus if we set $k=D-D^{\prime}$,

$$
\kappa_{D^{\prime}}=\frac{p^{2}}{4}\left[\tan \left(\frac{k \pi}{2 D}\right)-2 \tan \left(\frac{(k-1) \pi}{2 D}\right)+\tan \left(\frac{(k-2) \pi}{2 D}\right)\right] .
$$

Fixing the ratio $x=D^{\prime} / D$, and approximating the above as a second derivative, we finally get

$$
\kappa_{D^{\prime}}=\kappa_{E(x D)} \simeq \pi^{2} \frac{p^{2}}{8} \frac{1}{D^{2}} \tan (\pi(1-x) / 2)\left[1+\tan ^{2}(\pi(1-x) / 2)\right] .
$$

Thus the number of tiles in $\mathcal{A}_{E(x D)}$ is

$$
N_{E(x D)}=\alpha_{E(x D)} \phi_{D}(x)=2 D \kappa_{E(x D)} \phi_{D}(x) .
$$

For later reference we also calculate the average area of a tile in $\mathcal{A}_{E(x D)}$,

$$
\sigma(x)=\frac{\sum_{1 \leqslant i, j \leqslant E(x D)} \sin ^{2} \frac{(j-i) \pi}{D}}{\sum_{1 \leqslant i, j \leqslant E(x D)} \sin \frac{(j-i) \pi}{D}} \simeq \frac{\cos (2 \pi x)-1+2 \pi^{2} x^{2}}{8(\pi x-\sin \pi x)} .
$$

Finally, the fraction of tiles in the region $\mathcal{A}_{E(x D)}$ is

$$
\begin{aligned}
n_{E(x D)}= & \frac{N_{E(x D)}}{N_{T}}=\frac{N_{E(x D)}}{D^{2} p^{2} / 2} \\
\simeq & \frac{1}{2 D} \tan (\pi(1-x) / 2)\left[1+\tan ^{2}(\pi(1-x) / 2)\right] \\
& \times[\pi x-\sin \pi x],
\end{aligned}
$$

when $D$ goes to infinity, since the number of tiles in the whole tiling is $N_{T}=D^{2} p^{2} / 2$.

As expected, these fractions vanish but they can be added to get a non vanishing number of intersections. More precisely, let us fix a real 
number $0 \leqslant g \leqslant 1$ and let us compute the fraction of tiles which lie in regions where at least $E(g D)$ families of lines intersect:

$$
\begin{aligned}
\frac{1}{2}+\sum_{l=E(g D)}^{D-1} n_{l} \simeq & \frac{1}{2}+\frac{1}{2} \int_{0}^{1-g} d x \tan (\pi x / 2)\left[1+\tan ^{2}(\pi x / 2)\right] \\
& \times[\pi(1-x)-\sin \pi x] \equiv \frac{1}{2}+\frac{1}{2} f(1-g) .
\end{aligned}
$$

The above integral is a continuous function $f(g)$ for $g \in[0,1]$. It fulfills the required condition $f(0)=1$ (which means that the whole tiling contains a fraction 1 of tiles!). Moreover, $f(g)<1$ when $g>0$. Thus for any $h<1$, there exists a real number $g>0$ such that a fraction $h$ of the tiles lie in regions where at least $E(g D)$ families of lines meet. Since $g>0$, when $D$ goes to infinity, the number of families of lines also goes to infinity in such regions.

Moreover, Appendix B demonstrates that the local tilings in such regions are true large codimension ones. Hence everywhere in the above region, the local entropy tends to the free boundary entropy $\sigma_{\infty}$. A fraction $h$ of the tiles lie in regions where the local entropy per tile tends to $\sigma_{\infty}$ when $D \rightarrow \infty$. We get ${ }^{(11)}$ the expected relation (A.2) and we conclude that free and fixed boundary entropies coincide.

These arguments can also be extrapolated to finite $p$ tilings provided $D$ is large. Indeed, if $p$ is finite, a domain $\mathcal{K}_{D^{\prime}}$ can be very small and can contain very few tiles (and even no tile at all). Nevertheless in the previous demonstration, regions $\mathcal{A}_{E(x D)}$ with $x_{0} \leqslant x \leqslant x_{0}+\delta x$ can be put together into larger regions, to which all the previous arguments can be applied.

Finally we remark that the tiling $\mathcal{T}_{0}$ which was not assumed a priori to maximize the entropy functional indeed does so at the large $D$ limit. Therefore it should be close to generic and should give a good idea of the macroscopic structure of large $D$ generic tilings, in particular as far as the scaling laws of Section A.3 are concerned.

\section{A.2. Non-diagonal Tilings}

For large $p$ non-diagonal tilings with side lengths $k_{i}=x_{i} p$, where $\alpha \leqslant$ $x_{i} \leqslant \beta$, we use a particular tiling $\mathcal{T}_{0}^{\prime}$ which is a variation of $\mathcal{T}_{0}$. Its overall definition is the same except that the line spacing $l_{i}$ depends on the de Bruijn family $F_{i}$. We follow the main steps of the prior demonstration, in particular the calculation of the areas $\kappa_{D^{\prime}}$ or $\alpha_{D^{\prime}}$. 
We choose $l_{i}=p /\left(x_{i} p-1\right) \simeq 1 / x_{i}$ so that the width $l_{i}\left(k_{i}-1\right)=p$ of the family $F_{i}$ is independent of $i$ in the grid representation (Fig. 15, left). Therefore the areas $\alpha_{D^{\prime}}$ remain unchanged as compared to Section.

On the other hand, the density of intersections in each domain $\mathcal{K}_{D^{\prime}}$ will vary because of varying line spacing. In particular the area $\alpha_{0}$ of the unit cell now depends on $i$ and $j$ :

$$
\alpha_{0}(i, j)=\frac{l_{i} l_{j}}{\left|\sin \theta_{i j}\right|}=\frac{1}{x_{i} x_{j}\left|\sin \theta_{i j}\right|} .
$$

The number of intersections (A.6) becomes

$$
\tilde{Q}_{D^{\prime}}=\kappa_{D^{\prime}}\left(\sum_{1 \leqslant i<j \leqslant D^{\prime}} x_{i} x_{j} \sin \frac{(j-i) \pi}{D}\right) \leqslant \beta^{2} Q_{D^{\prime}}
$$

Henceforth, the new numbers of tiles in the crown $\mathcal{A}_{D^{\prime}}$ and in the whole tiling satisfies $\tilde{N}_{D^{\prime}} \leqslant \beta^{2} N_{D^{\prime}}$ and $\tilde{N}_{T} \geqslant \alpha^{2} N_{T}$. If we set again $D^{\prime}=E(x D)$, the new fraction of tiles in $\mathcal{A}_{D^{\prime}}$ satisfies

$$
\tilde{n}_{E(x D)}=\frac{\tilde{N}_{E(x D)}}{\tilde{N}_{T}} \leqslant \frac{\beta^{2}}{\alpha^{2}} n_{E(x D)}
$$

As a consequence, the outer crowns with small effective dimensionality $D^{\prime}$, which had a vanishingly small contribution to the total number of tiles in the previous section, will again be negligible in the present case.

To finish the proof, we must check that the remainder of the tiling has a local entropy equal to $\sigma_{\infty}$. The proof is explicited in Appendix B in the diagonal case but can easily be adapted to the present case, leading to

$$
\frac{n_{i j}^{\prime}}{\left(n^{\prime}\right)_{i j}^{*}} \geqslant \frac{\alpha^{2}}{\beta^{2}} \frac{6}{\pi^{2}},
$$

where the notations are defined in appendix B. Even though the lower bound on tile fractions now depends on $\alpha$ and $\beta$ because we are dealing with a nondiagonal case, it remains finite. 


\section{A.3. Scaling Law for the Effective Dimensionality}

We derive the effective dimensionality $D^{\prime}=D_{\text {eff }}(r)$ in the tiling $\mathcal{T}_{0}$ of side length $p$ at the large $D$ limit. Here $0<r<R$ is the distance from the tiling center $O$ and $R \simeq p D / \pi$ is the radius of the tiling. Assume that in a large $D$ tiling, a region of effective dimensionality $D_{\text {eff }}$ is an annulus $\mathcal{A}_{D_{\text {eff }}}$ of center $O$, the tiling center, and of radii $r$ and $r+\delta r$. Hence $D_{\text {eff }}(r+$ $\delta r)=D_{\text {eff }}(r)-1$ (the effective dimensionality decreases with increasing $r$ ), that is to say

$$
\delta r \frac{d}{d r} D_{\text {eff }}(r)=-1
$$

Now we need to compute $\delta r$. Following Appendix (A.1) we write $x=$ $D_{\text {eff }} / D$. If we knew the area $s(x)$ of $\mathcal{A}_{D_{\text {eff }}}$ in the tiling metric, then we could extract $\delta r$ from $s(x)=2 \pi r \delta r$, in order to write

$$
s(x) \frac{d}{d r} D_{\text {eff }}(r)=-2 \pi r .
$$

But we can easily extract $s(x)$ from Eqs. (A.14) and (A.15) for the number and area of tiles in $\mathcal{A}_{E(x D)}$ to find

$$
\begin{aligned}
s(x)= & \frac{D p^{2}}{32} \tan (\pi(1-x) / 2)\left[1+\tan ^{2}(\pi(1-x) / 2)\right] \\
& \times\left[\cos (2 \pi x)-1+2 \pi^{2} x^{2}\right] .
\end{aligned}
$$

It is convenient to introduce dimensionless variables which remove the divergences for large $D$ and $p$. Specifically, we introduce $\hat{r} \equiv r / R$ and $\hat{s}(x) \equiv s(x) /\left(D p^{2}\right)$, and we recall that $D_{\text {eff }}(r) / D=x$ and $R=p D / \pi$ to obtain

$$
\hat{s}(x) \frac{d}{d \hat{r}} x=-\frac{2}{\pi} \hat{r},
$$

which is completely equivalent to Eq. (A.22).

We can solve Eq. (A.25) by direct integration to obtain the radius $\hat{r}$ corresponding to a given effective dimension $x$, 


$$
\begin{aligned}
& \hat{r}^{2}(x)-1=-\pi \int_{0}^{x} s\left(x^{\prime}\right) d x^{\prime} \\
&=\frac{1}{16}((-14+2 \cos (\pi x) \\
&\left.+\pi x \csc ^{2}\left(\frac{\pi x}{2}\right)(\pi x+2 \sin \pi x)\right)
\end{aligned}
$$

or we can invert the solution and obtain (after returning to unscaled variables)

$$
D_{\text {eff }}(r)=D \gamma(r / R)
$$

with $\gamma(\hat{r})$ the inverse of $\hat{r}(x)$. Although the solution in Eq. (A.26) cannot be inverted in closed form, we can expand it for small $x$ to find values of $r$ close to the boundary $R$. Defining the small quantity $\epsilon=1-r / R$, we have

$$
\epsilon=\frac{\pi^{2} x^{2}}{24}+O\left(x^{4}\right)
$$

then by reversion of series obtain

$$
\gamma(r / R)=x \approx \frac{\sqrt{24}}{\pi} \sqrt{\epsilon}
$$

Apparently, the effective dimensionality varies rapidly near the boundary.

We also use the notion of effective codimension $c_{\text {eff }}=D_{\text {eff }}-2$. At large $D$, one has

$$
\frac{c_{\mathrm{eff}}}{D-2} \simeq \frac{D_{\mathrm{eff}}}{D}=\gamma(r / R)
$$

All the quantities expressed as functions of $x$ are also functions of $r / R$. For example, the vertex density

$$
d(x)=\frac{1}{\sigma(x)} \simeq \frac{2}{\pi x}=\frac{2}{\pi \gamma(r / R)} \simeq \frac{1}{\sqrt{6(1-r / R)}}=d(r / R)
$$

at small $x$, i.e. when $r$ close to $R$. This scaling compares well with numerical data in Section 4. 


\section{APPENDIX B: REGULARITY OF LARGE CODIMENSION FIXED BOUNDARY TILINGS}

We check here that in each region $\mathcal{A}_{D^{\prime}}$ of the diagonal tiling $\mathcal{T}_{0}$ the local entropy per tile tends to the free boundary one $\sigma_{\infty}$ as $D \rightarrow \infty$. As discussed in paper $I^{(1)}$, a sufficient condition is that tile fractions should be "bounded" on $\mathcal{A}_{D^{\prime}}$. This means that all local tile fractions $n_{i j}$ have the same order of magnitude as the strain-free ones $n_{i j}^{*}$. In other words, there exist a finite constant $a>0$ such that

$$
n_{i j} \geqslant a n_{i j}^{*}
$$

for all $i$ and $j$. We want to calculate the tile fractions $n_{i j}^{\prime}$ in $\mathcal{A}_{D^{\prime}}$ and compare them to the strain-free ones. Recall that in a $D \rightarrow 2$ tiling,

$$
n_{i j}^{*}=C \sin \left|\pi \frac{j-i}{D}\right|
$$

where the constant $C$ comes from the normalization relation $\sum n_{i j}^{*}=1$.

As in Appendix A, we set $D^{\prime}=E(x D)$ where $D$ is large and $x>0$ is finite. The main difficulty in this appendix comes from the fact that in the region $\mathcal{A}_{D^{\prime}}$ under consideration, not all tile species occur. Without loss of generality, we assume that only the tiles with indices $i, j=1, \ldots, D^{\prime}$ exist. These tiles appear in the region $\mathcal{A}_{E(x D)}$ with the fractions (B.2), but with a different normalization constant because only some of them appear. The normalization relation now reads

$$
\sum_{1 \leqslant i<j \leqslant D^{\prime}} n_{i j}^{\prime}=1
$$

At the large $D$ limit, this sum can be replaced by an integral and we get the tile fractions in $\mathcal{A}_{E(x D)}$ :

$$
n_{i j}^{\prime}=\frac{1}{\left(D^{\prime}\right)^{2}} \frac{\pi^{2} x^{2}}{\pi x-\sin (\pi x)} \sin \left|x \pi \frac{j-i}{D^{\prime}}\right| .
$$

Now we check that this tiling has bounded local fractions of tiles when it is considered as a $D^{\prime} \rightarrow 2$ tiling. We need to compare the above $n_{i j}^{\prime}$ to the corresponding quantities in an unstrained $D^{\prime} \rightarrow 2$ tilings, in other words to

$$
\left(n^{\prime}\right)_{i j}^{*}=\frac{\pi}{\left(D^{\prime}\right)^{2}} \sin \left|\pi \frac{j-i}{D^{\prime}}\right|
$$


By convexity of the sin function on the interval $[0, \pi]$, we have: $\sin \mid x \pi(j-$ i) $/ D^{\prime}|\geqslant x \sin | \pi(j-i) / D^{\prime} \mid$, and

$$
\frac{n_{i j}^{\prime}}{\left(n^{\prime}\right)_{i j}^{*}} \geqslant \frac{x}{\pi} \frac{\pi^{2} x^{2}}{\pi x-\sin (\pi x)} \geqslant \frac{6}{\pi^{2}},
$$

which achieves the proof: $a=6 / \pi^{2}$ in condition (B.1). From the results of paper $\mathrm{I}$, ${ }^{(1)}$ we conclude that in such a region $\mathcal{A}_{D^{\prime}}$, the local entropy per tile equals the free boundary value $\sigma_{\infty}$ when $D \rightarrow \infty$.

\section{ACKNOWLEDGMENTS}

We thank Chris Henley and Pavel Kalugin for useful discussions. This research is supported in part by the National Science Foundation under grants DMR-0111198 and INT-9603372 and by the CNRS.

\section{REFERENCES}

1. N. Destainville, M. Widom, R. Mosseri, and F. Bailly, Random tilings of high symmetry: I. Mean-field theory, J. Stat. Phys. 120(5/6):799-835 (2005).

2. V. Elser, Phys. Rev. Lett. 54:1730 (1985).

3. N. G. de Bruijn, Ned. Akad. Wetensch. Proc. A84:39 (1981); J. Phys. France 47:C3-9 (1986).

4. D. Shechtman et al., Phys. Rev. Lett. 53:1951 (1984).

5. D. Levine and P. J. Steinhardt, Phys. Rev. Lett. 53:2477 (1984).

6. C. L. Henley, in D. P. Di Vincenzo and P. J. Steinhart eds. Quasicrystals, the State of the Art (World Scientific, 1991), p. 429.

7. V. Elser, J. Phys. A 17:1509 (1984).

8. G. H. Wannier, Phys. Rev. 79:357 (1950); Phys. Rev. B 7:5017 (1973).

9. D. Grensing and G. Grensing, J. Math. Phys. 24:620 (1983); D. Grensing, I. Carlsen and H.Chr. Zapp, Phil. Mag. A 41:777 (1980).

10. H. Cohn, M. Larsen, and J. Propp, New York J. of Math. 4:137 (1998); H. Cohn, R. Kenyon, and J. Propp, J. Amer. Math. Soc. 14:297 (2001).

11. N. Destainville, J. Phys. A: Math. Gen 31:6123 (1998).

12. M. Widom, N. Destainville, R. Mosseri, and F. Bailly, in Proceedings of the 6th International Conference on Quasicrystals (World Scientific, Singapore, 1997).

13. N. Destainville, M. Widom, R. Mosseri, and F. Bailly, Mat. Sci. Eng. A 294-296:409 (2000).

14. M. Widom, R. Mosseri, N. Destainville, and F. Bailly, J. Stat. Phys. 109:945 (2002).

15. N. Destainville, R. Mosseri, and F. Bailly, J. Stat. Phys. 102:147 (2001).

16. R. Mosseri and F. Bailly, Int. J. Mod. Phys. B 6 \& 7:1427 (1993).

17. N. Destainville, R. Mosseri, and F. Bailly, J. Stat. Phys. 87:697 (1997).

18. D. M. Knuth, Axioms and Hulls, in Lect. Notes in Computer Sci. 606:35 (1992).

19. A. Björner, M. Las Vergnas, B. Sturmfels, N. White, and G.M. Ziegler Oriented Matroids (Cambridge University Press, Cambridge, 1993).

20. R. Kenyon, Algorithmica 9:382 (1993). 
21. S. Elnitsky, J. Combinatorial Theory A 77: 193-221 (1997).

22. G. D. Bailey Tilings of Zonotopes: Discriminental Arrangements, Oriented Matroids, and Enumeration, Ph. D. Thesis (University of Minnesota, 1997).

23. W. Li, H. Park, and M. Widom, J. Stat. Phys. 66:1 (1992).

24. M. E. J. Newman and G. T. Barkema Monte Carlo Methods in Statistical Mechanics (Clarendon Press, Oxford, 1999).

25. K. J. Strandburg, L.-H. Tang, and M. V. Jaric, Phys. Rev. Lett. 63: 314 (1989); L. J. Shaw, V. Elser, C. L. Henley, Phys. Rev. B 43:3423 (1989); L.-H. Tang, Phys. Rev. Lett. 64:2390 (1990); M. Oxborrow and C. L. Henley, Phys. Rev. B 48:6966 (1993); F. Gahler, Proc. ICQ5; 236 (1995); D. Joseph and M. Baake, J. Phys. A 29:6709 (1996).

26. M. Luby, D. Randall, and A. Sinclair, SIAM J. of Comp. 31:167 (2001); N. Destainville, Phys. Rev. Lett. 88:30601 (2002).

27. Strictly speaking, should this fan be exactly regular, there would be multiple intersections, for example at its very center. To avoid this difficulty, each family must be slightly shifted by a random distance much smaller than the interline separation. Such shifts have been performed in the Fig. 14, even though it might not be clear because of resolution.

28. A rectangular kite has two right angles between its unequal sides. 